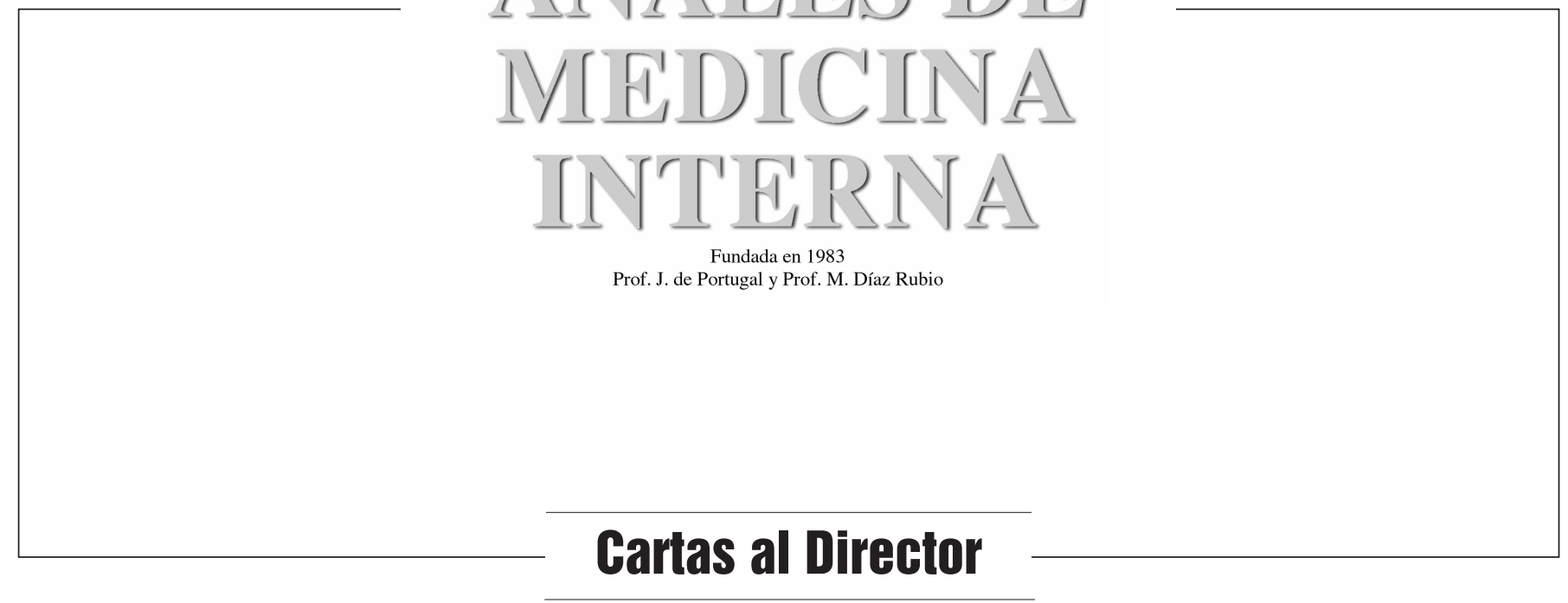

\section{Importancia de las apoproteínas A1 y B como marcadores de riesgo cardiovascular}

\section{Sr. Director:}

Las enfermedades cardiovasculares constituyen un problema de salud pública de primer nivel en la edad adulta. La enfermedad de las arterias coronarias constituye una de las principales causas de muerte y de consumo de productos sanitarios en los países desarrollados.

España es una país mediterráneo que tiene una baja mortalidad coronaria, sin embargo en algún estudio reciente se ha mostrado que la prevalencia de factores de riesgo no es significativamente diferente a la que se puede encontrar en otras sociedades occidentales que tienen una mortalidad mucho más elevada (1), lo que indica la existencia de una evidente paradoja entre ambas realidades, mortalidad relativamente baja y prevalencia de factores de riesgo relativamente elevada (2).

Debido a que las alteraciones ateroscleróticas tienen su origen en edades relativamente tempranas, sería del máximo interés identificar marcadores bioquímicos en edades tempranas de la vida, para realizar un tratamiento corrector de las alteraciones perjudiciales en el patrón lipídico.

Evidentemente hay un gran número de sustancias a considerar, pero de un modo breve, nos parece interesante realizar unas reflexiones sobre las apoproteínas A1 y B, como marcadores de riesgo cardiovascular.

Las apoproteínas permiten el transporte de lípidos en el compartimiento intravascular y extravascular.

Dentro de los diferentes tipos de apoproteínas vamos a mencionar fundamentalmente a las apo B y la apo A1.

La capacidad de estimular el transporte de lípidos por parte de las HDL, parece ser exclusiva de la apoA1 (3). Este proceso permite que las HDL realicen un lavado rápido y eficiente del exceso del colesterol y su almacenamiento como éster de colesterol. El transporte del colesterol por las HDL desde las células extrahepáticas hacía el hígado para ser eliminado por la bilis, es el mayor responsable del efecto protector de esta lipoproteína contra la aterosclerosis $(3,4)$.

En la conversión de VLDL a LDL, la apoB sufre un cambio conformacional que permitirá la unión de las LDL a su receptor y su salida de la circulación.
Se han relacionado concentraciones elevadas de apo B con estados de hiperinsulinemia y resistencia a la insulina (5-7).

En niños y adolescentes obesos se ha comunicado en los casos en los que los niveles de colesterol total se encuentra dentro de los límites normales, las relaciones colesterol total/HDL-colesterol, LDL-colesterol/ HDL-colesterol y apoB/apo A1, suelen estar elevadas con respecto a los adolescentes no obesos, lo que mostraría una disminución relativa de las HDL-colesterol $(5,6)$.

Los niveles de apoproteínas B y A1 se miden por inmunoturbimetría, siendo los valores de referencia para apo A1 de 115 a $220 \mathrm{mg} / \mathrm{dl}$ y para la apoB de 60 a $138 \mathrm{mg} / \mathrm{dl}$ (6).

Niveles elevados de apo A1 se asocian negativamente con el índice de obesidad, un índice de riesgo cardiovascular bajo y positivamente con los niveles de HDL-colesterol.

A su vez los niveles elevados de apo B, se relacionan de modo positivo con los niveles de colesterol total y con los de LDLcolesterol, lo que indicaría un mayor riesgo cardiovascular. Este grupo de pacientes muestra a menudo, niveles de triglicéridos más elevados, niveles más elevados de insulina basal y menores de HDL-colesterol.

Incrementos moderados en los triglicéridos pueden alterar el contenido de colesterol de las partículas de HDL.

Diversos estudios han demostrado que existen subespecies de estas lipoproteínas con distintas propiedades bioquímicas y metabólicas(8), así se ha descrito una subespecie de LDL de menor tamaño y más densa, denominada fenotipo aterogénico, que esta asociado con niveles elevados de triglicéridos VLDL y de apo B y niveles bajos de HDL-colesterol y de apo A1. Es evidente que todas estas moléculas por separado están relacionadas con la aparición precoz de la enfermedad arterial coronaria (9).

Incluso algún estudio sugiere que más importante que los niveles de colesterol de la HDL, es su composición en el contenido de la apoproteína A1 (9), lo que mostraría el interés. de estas apoproteínas.

De todas formas, estos biomarcadores no están de momento indicados en la práctica clínica habitual, siendo preciso realizar un mayor número de estudios clínicos randomizados, que analicen los cambios en los niveles de estas apolipoproteinas en las distintas situaciones clínicas (10).

F. M. Sánchez, M. I. Albo Castaño, S. Casallo Blanco, A. Vizuete Calero, L. de Matías Salces 
Servicio de Medicina Interna. Hospital Nuestra Señora del Prado. Talavera de la Reina. Toledo

1. Ballesteros Pomar MD, Rubio Herrero MA, Gutiérrez Fuentes JA, Gómez Guerique JA, Gómez de la Cámara A, Pascual O, et al. Dietary, Habits and Cardiovascular Risk in Spanisch Population. The DRECE Study(II). Micronutrient Intake. Ann Nutr Metab 2000; 44: 177-82.

2. Gómez de la Cámara A, Rubio-Herrera MA, Gutiérrez Fuentes JA, Gómez Guerique JA, del Campo J, Jurado Valenzuela C, et al. Mortalidad en el estudio DRECE-III ( Dieta y riesgo de Enfermedades Cardiovasculares en España). Clin Invest Arterioscl 2007; 19 (Supl.): 5: 20-3.

3. Oram JF, Yokoyama S. Apolipoprotein mediated removal of celular colesterol and phospholipids. J Lipid Res 1996; 37: 2473-91.

4. Thompson A, Danesh J. Association between apolipoprotein B, apolipoprotein AI, the apolipoprotein $\mathrm{B} / \mathrm{AI}$ ratio and coronary heart disease: a literature-based meta-analysis of prospective studies. J. Intern Med 2006; 259: 481-92.
5. Luc G, Bard JM, Ferrieres J, Evans A, Amouyel P, Arveiler D. Values of HDL cholesterol, apolipoprotein A1 and Lipoprotein A1 and Lipoprotein AI/AII in prediction of coronary heart disease. The PRIME Study. Atheroscler Thromb Vasc Biol 2002; 22: 1155-60.

6. Marcovina S, Packard CJ. Measurement and meaning of apolipoprotein A1 and apolipoprotein B plasma levels. J Intern Med 2006; 259: 437 46.

7. Reaven GM, Chen YD, Leppesen J, Maheux P, Krauss RM. Insulin resistance and hyperinsulinaemia in individuals with small dense low density lipoproteins particles. J Clin Invest 1993; 92: 141-2.

8. Bertiere MC, Fumeron F, Regaud D, Apfelbaum M, Guard-Globa A. Low high density lipoproteins 2 concentration in obese male subjects. Atherosclerosis 1988; 73: 57-61

9. Koening W. Atherosclerosis lab risk markers today: Research and clinical application. Clin Invest Arterioscl 2007; 19 (Supl. 5): 39-42.

10. Koening W, Khuseyinova N. Biomarkers of atheroesclerotic plaque instability and rupture. Arterioescl Thromb Vasc Biol 2007; 27: 15-26. 